Aboul-Ella Hassanien, Ajith Abraham, and Francisco Herrera (Eds.)

\title{
Foundations of Computational Intelligence Volume 2
}

Approximate Reasoning 
Prof. Aboul-Ella Hassanien

Cairo University

Faculty of Computers and Information

Information Technology Department

5 Ahmed Zewal St.

Orman, Giza

E-mail: Aboitcairo@gmail.com

http://www.fci.cu.edu.eg/abo/

\section{Prof. Ajith Abraham}

Machine Intelligence Research Labs

(MIR Labs)

Scientific Network for Innovation and

Research Excellence

P.O. Box 2259

Auburn, Washington 98071-2259

USA

E-mail: ajith.abraham@ieee.org
Prof. Francisco Herrera

Soft Computing and Intelligent Information

Systems

Department of Computer Science and

Artificial Intelligence

ETS de Ingenierias Informática y de

Telecomunicación

University of Granada

E-18071 Granada

Spain

E-mail: Herrera@decsai.ugr.es

ISBN 978-3-642-01532-8

e-ISBN 978-3-642-01533-5

DOI 10.1007/978-3-642-01533-5

Studies in Computational Intelligence

ISSN 1860949X

Library of Congress Control Number: Applied for

(C) 2009 Springer-Verlag Berlin Heidelberg

This work is subject to copyright. All rights are reserved, whether the whole or part of the material is concerned, specifically the rights of translation, reprinting, reuse of illustrations, recitation, broadcasting, reproduction on microfilm or in any other way, and storage in data banks. Duplication of this publication or parts thereof is permitted only under the provisions of the German Copyright Law of September 9, 1965, in its current version, and permission for use must always be obtained from Springer. Violations are liable to prosecution under the German Copyright Law.

The use of general descriptive names, registered names, trademarks, etc. in this publication does not imply, even in the absence of a specific statement, that such names are exempt from the relevant protective laws and regulations and therefore free for general use.

Typeset \& Cover Design: Scientific Publishing Services Pvt. Ltd., Chennai, India.

Printed in acid-free paper

987654321

springer.com 


\title{
A Bayesian Solution to the Modifiable Areal Unit Problem
}

\author{
C. Hui
}

Centre of Excellence for Invasion Biology, Department of Botany and Zoology, University of Stellenbosch, Private Bag X1, Matieland 7602, South Africa chui@sun.ac.za

Summary. The Modifiable Areal Unit Problem (MAUP) prevails in the analysis of spatially aggregated data and influences pattern recognition. It describes the sensitivity of the measurement of spatial phenomena to the size (the scale problem) and the shape (the aggregation problem) of the mapping unit. Much attention has been recieved from fields as diverse as statistical physics, image processing, human geography, landscape ecology, and biodiversity conservation. Recently, in the field of spatial ecology, a Bayesian estimation was proposed to grasp how our description of species distribution (described by range size and spatial autocorrelation) changes with the size and the shape of grain. This Bayesian estimation (BYE), called the scaling pattern of occupancy, is derived from the comparison of pair approximation (in the spatial analysis of cellular automata) and join-count statistics (in the spatial autocorrelation analysis) and has been tested using various sources of data. This chapter explores how the MAUP can be described and potentially solved by the BYE. Specifically, the scale and the aggregation problems are analyzed using simulated data from an individual-based model. The BYE will thus help to finalize a comprehensive solution to the MAUP.

\section{Introduction}

Spatial patterns in the natural world are hardly random, from the cloud of atoms to the distribution of species. Relating such patterns across scales has been argued as the central problem in all of science 1. In physics, Bose-Einstein condensation trys to describe how numerous atoms are distributed in lattices with changing sizes [2]. In ecology, alpha and beta diversity shows the scale sensitivity of species richness $\underline{3}$. The species-area relationship 4, 5, and the scaling pattern of species distribution (measured by occupancy or occurrence) [6] attract much attention from ecologists. In the analysis of those patterns across scales (called scaling patterns), a severe statistical

A.-E. Hassanien et al. (Eds.): Foundations of Comput. Intel. Vol. 2, SCI 202, pp. 175-196. springerlink.com

(c) Springer-Verlag Berlin Heidelberg 2009 
problem emerges, i.e. how the measurements of those spatial patterns depend on the scales in question, named the Modifiable Areal Unit Problem (hereafter MAUP) [7].

MAUP is a potential source of error that can affect spatial studies which utilize aggregate data sources [8]. To describe a spatial phenomenon, we normally transform it into a raster format, like a digital picture, with each minimal unit called grain [9]. As the the grain changes, so does the measurement of the spatial pattern (e.g. spatial variance [10]). MAUP consists of two parts: the scale problem and the aggregation problem (also called the zoning problem), which are caused by the changing of the size and the shape of grain, respectively [7]. Although there is hitherto no complete solution to MAUP [11, studies on the scale problem prevail in the spatial analysis of ecology. The non-random distribution of species in its spatial habitat has long been studied (e.g. [12]). This non-randomness arises from the spatial heterogeneity of the habitat, the nonlinear essence of species life history (e.g. dispersal strategy and density dependence), as well as interspecific interactions. Because most species' distributions are not random but aggregated (or over-dispersed, clustered, clumpy, autocorrelated, contagious, patchy, etc.), finding a consistent description of the spatial structure of species distributions becomes a priority in ecological research. On the other hand, the aggregation (zoning) problem has recieved little attention in ecology, with only a little from human geography 8.

Since MAUP only affects non-random spatial data [7, different methods and aggregation indices have been invented to grasp the characteristics of this non-randomness in nature. According to Li and Reynolds' 13 introduction, Wiens [12] categorised the spatial heterogeneity into four forms: spatial variance, patterned variance, compositional variance and locational variance. Based on the standard geographic system [14, Perry et al. 15] divided the types of spatial data into point- and area-referenced data. Different spatial statistics focus on different types of spatial data [16. Clarification of the concept of aggregation as well as the scaling pattern of the aggregation indices plays an important role in the unification of the theories in macroecology and spatial ecology.

In the development of spatially semi-explicit indices, belonging to the Local Indicators of Spatial Autocorrelation (LISA) statistics [17], progress was made through linking pair approximation and join-count statistics [6, 18]. Pair approximation is a moment approximation method for describing spatial patterns, introduced from statistical physics [19, and has been widely employed in the analysis of spatial patterns from cellular automata (e.g. 20]). Join-count statistics is the first step in describing real spatial patterns by giving the status of a focal sample's neighbors (adjacent patches) [21, 22. It is possible that the combination of these two methods can address a simplified MAUP (i.e. in presence-absence format), and eventually provide a comprehensive framework for solving the MAUP in the future. 


\section{Models}

\subsection{Modifiable Areal Unit Problem}

\section{Literature Review}

MAUP first originated from the analysis of scale effects in census data 23], and was formally demonstrated in 1980's [7. Jelinski and Wu [24] indicated that the MAUP arises from the fact that areal units are usually arbitrarily determined and "modifiable", in the sense that they can be aggregated to form units of different sizes or spatial arrangements. Openshaw [7 divided the MAUP into two sub-problems (scale and aggregation). The scale problem is "the variation in results that may be obtained when the same areal data are combined into sets of increasingly larger areal units of analysis"; the aggregation (zoning, zonation) problem is "any variations in results due to alternative units of analysis where the number of units, is constant".

Research on the MAUP has tended to focus on the meaurement of spatial structure using different indices and statistics (e.g. 25]). Dorling [26] highlighted difficulites such as the MAUP in preventing the development of visual palliatives and pointed out the necessity of using cartograms in place of conventional choropleth maps for aggregated distributions. Amrhein [27] concluded that the effects of MAUP vary with the statistic calculated. Even though means and variances are resistant to aggregation effects, regression coefficients and correlation statistics exhibit dramatic effects. Amrhein [27] reminded us that the world of spatial analysis is not entirely well-behaved due to the MAUP. With the increased awareness of MAUP in Geographic Information Systems (GIS) based spatial analysis in physical geography [28, landscape ecology 24] and social, urban management 29, 30, case studies on scaling patterns flourished. To list a few, Lery 31] performed a risk analysis for foster care entry at three spatial scales. Sexton et al. 32 examined spatial effects on analytical results related to causal inference and disease clustering. These studies recall Levin's [1] emphasis in science. In the meantime, traditional indices and statistics, as well as a wide range of new methods have been tested for their robustness and senstivity towards the MAUP (e.g. [33, 34)). As the MAUP was only formally termed after Openshaw in the 80's, the studies on MAUP, as reviewed here, were largely descriptive. A full understanding even of a simplified MAUP will help to put the efforts into a right research direction, which is the focus of this work.

\section{Presence-Absence MAUP}

In spatial analysis, one often encounters raster data, e.g. atlas and digital pictures, in which the presence-absence (binary) format is prevalent. For example, the .., , .... ..., 35 presents, for each species, a presence-absence map with a square $15 ' \times 15^{\prime}$ grain, showing the distribution of a particular species during the sampling period. The prevalence of 


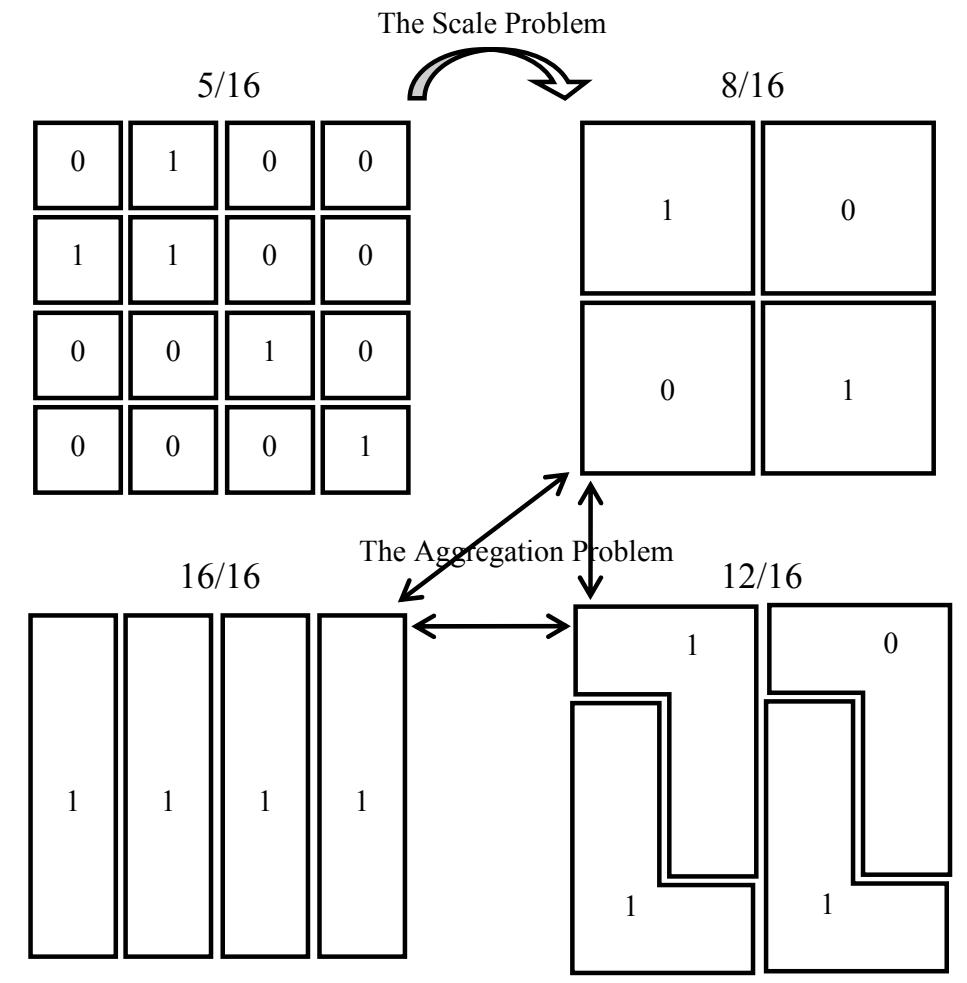

Fig. 1. A simplified illustration of the scale and aggregation problems in the modifiable areal unit problem (MAUP)

presence-absence data is due to the fact that it is the easiest and quickest representation, which can be provided for a large-scale region at relatively low cost 36. Spatial niche modelling (such as the climatic envelope; 37]) can also provide a quick, large-scale presence-absence map for a species distribution in landscape ecology. Lattice models also provide a huge amount of binary data from theoretical and experimental studies (e.g. [38, 39]). However presence-absence data is susceptible to the MAUP, and a framework for overcoming this downfall is proposed in the pages to follow.

To clearly understand the MAUP, an illustrative example is given in figure 1 using such binary data. In figure 1 , the original grain is 1/16 and the occupancy (i.e. the proportion of occupied cells) is $5 / 16$. If we keep the shape of the grain unchanged but combine the four nearest neighbors into a new grain, we have a new grain size of $4 / 16$. Now the MAUP occurs. We notice that the occupancy is not $5 / 16$ anymore, but $8 / 16$. This deviation between $5 / 16$ and $8 / 16$ is caused by the MAUP and is termed the scale problem. If we let the grain size remain $4 / 16$, but change the shape of the grain (as shown in figure 1) from square to rectangular (with the width to length ratio being 1:4) and L-shaped, we find 
that the occupancy will also change (16/16 for rectangle grain and 12/16 for L-shaped grain). Such a difference is caused by the shape of the grain, called the aggregation problem (also called the zoning problem). A solution to this simplified MAUP (i.e. in a presence-absence version) should indicate how the occupancy will change with grain size as well as shape.

\subsection{Individual Based Model}

A standard plant population presence-absence data from a spatially explicit individual-based model (IBM) was generated. IBM is a typical model in ecological research, which has been widely used in the simulation of mixed ecosystems, fish, mammals, birds, insects, marine invertebrates, arachnids, bacteria, and other non-species-specific models (e.g. ATLSS by Donald DeAngelis et al. http://atlss.org/).

An individual in this simulation can be seen as a hermaphrodite perennial bush. A number of $n$ seeds $(n=10)$ will be dispersed around the parent, which obeys an exponential distribution $p(x)=\lambda e^{-\lambda x}(x$ is the distance between the seed and the parent; $1 / \lambda$ gives the mean distance of seeds to parent, $0.01 \leq \lambda \leq$ 5.5 ; here we chose $\lambda=1$ ) with a randomly chosen direction $\theta\left(0^{\circ} \leq \theta \leq 360^{\circ}\right)$. The individuals that can produce seeds are randomly chosen with a probability of $c(c=0.25)$ within the mature adults (those whose age is older than one year). The seed can only have a chance to grow to a seedling if there are no other plants within a certain distance $d(d=0.2)$ (due to the overcrowding effect or other density-dependent mechanisms). During each time step, individuals also suffer a probability of death (mortality) $e(e=0.1)$.

The simulation was done in a $50 \times 50$ extent of a two-dimensional homogeneous space (figure 2A) with periodic boundary (to exclude the edge effect), which means the maximal number of individuals is 19,894 . This model also generated similar results as other individual-based models, such as the forest growth simulators JABOWA, FORET, and SORTIE [0, but it is distinguished from other lattice simulations (cellular automata; e.g. [41, 42]) by the fact that the individuals were not constrained to grids (or discrete in space), rather each one had its own coordinates. The two-dimensional space was then divided into a patch network (figure $2 \mathrm{~B}$ ). A patch with at least one individual was marked as presence (black in figure 2); while an empty one was marked as absence (white). The occupancies of figure $2 \mathrm{~B}, \mathrm{C}$ and $\mathrm{D}$ are $0.2794,0.6325$ and 0.65 , respectively. Specifically, we want to have a formula to predict the occupancies in figure $2 \mathrm{C}$ and $\mathrm{D}$ using the occupancy of figure $2 \mathrm{~B}$. This formula should not only grasp the difference in occupancies across scales (the scale problem) but also the difference for the same grain size (the aggregation problem).

\subsection{Local Indicators of Spatial Autocorrelation}

A specific field exists in ecology, namely spatial analysis, that focuses on the non-random, aggregated (contagious) spatial distributions of species [4], 
A
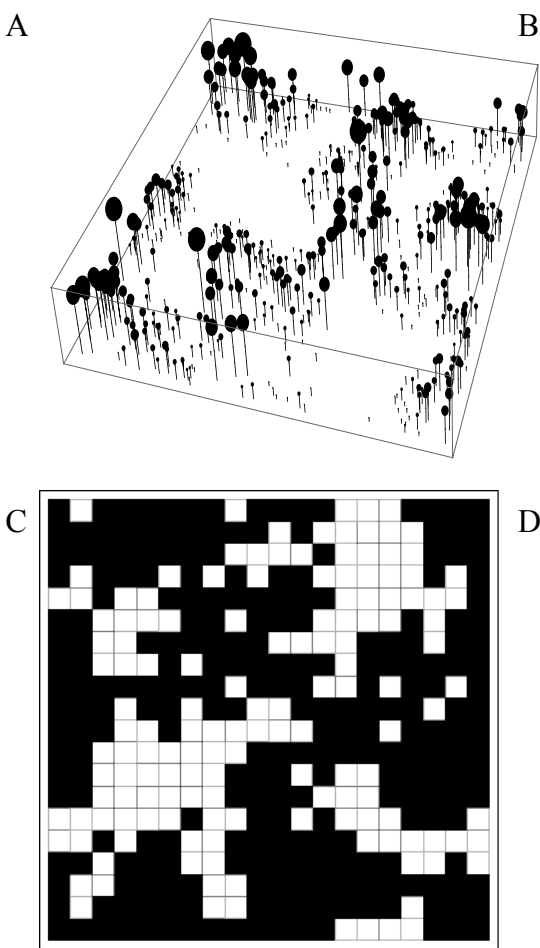

$\mathrm{B}$

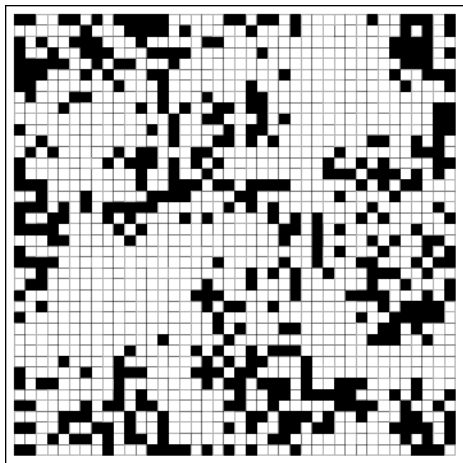

$\mathrm{D}$

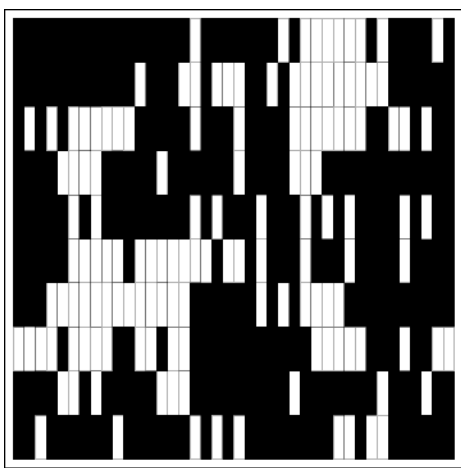

Fig. 2. The spatial pattern generated from the individual-based model. $\boldsymbol{A}$, A total of 541 individuals were generated after 20 time steps. $\boldsymbol{B}$, The presence-absence maps for the resolution of $40 \times 40$ grains. $\boldsymbol{C}$, The map for $20 \times 20$ grains. $\boldsymbol{D}$, The mape for $40 \times 10$ grains. See text.

i.e. the spatial heterogeneity of species distributions. Two factors can cause such phenomena of non-randomness in nature: spatial heterogeneity in habitat 44 and the nonlinearity of biological processes, such as density dependent growth and dispersal rates [45. The spatial heterogeneity caused by the IBM above belongs to the latter scenario. In the past several decades, different aggregation indices and measures have been developed focusing on different types of data in the spatial analysis [15, 16]. Depending on the degree of spatial information incorporated in the measure, three categories of spatial statistics can be further distinguished, i.e. spatially implicit, semi-explicit and explicit. Spatial implicit indices measure the statistical heterogeneity based on the mean and variance of the number of individuals in samples (cells) and were widely used in early literature, such as the coefficient of diffusion [46], Morisita's $I_{M}$ 47], Lloyd's $I_{L}$ [48, the clumping parameter $k$ in the negative binomial distribution [49] and the exponent $b$ in Taylor's power law [10]. With the focus of species distributions shifting from concerns of statistical heterogeneity to real spatial structure, spatially semi-explicit (spatial 
autocorrelation) indices were developed. These spatially semi-explicit measures describe patterned variance 12 and are formally known as local indicators of spatial autocorrelation (LISA) statistics 16. Indices, to list a few, include Ripley's $K$ function [50], Moran's $I$ [51] and correlograms [52. Most recently, spatially explicit indices were also developed based on the distance that would be required to move individuals to achieve a random distribution. One commonly used measure is Perry's [53, 54] spatial analysis by distance indices (SADIE).

The reasons for choosing a specific LISA index in this chapter are threefold. First, spatially implicit indices in fact do not depict the spatial structure of species distribution, and thus can not be used in the spatial scaling analysis that is related to the MAUP. Second, spatially explicit indices still rely largely on computer programming and thus make mathematical analysis difficult or impossible. Finally, the local spatial structures that are described by the LISA statistics reflect distance (or scale) dependent patterns, which are analogous to the scaling issue of the MAUP. It is exactly for this reason that LISA statistics are suitable here. Normally, a LISA index would have two versions: global and local versions. For example, the global index of the widely used Moran's I 43, 51] is,

$$
I(d)=\frac{1}{W(d)} \frac{\sum_{i=1, i \neq j}^{n} \sum_{j=1, j \neq i}^{n} w_{i j}(d)\left(x_{i}-\bar{x}\right)\left(x_{j}-\bar{x}\right)}{\frac{1}{n} \sqrt{\sum_{i=1}^{n}\left(x_{i}-\bar{x}\right)^{2}}}
$$

where $w_{i j}(d)(=1$ or 0$)$ is the distance class $d$-connectivity between cell (sample) $i$ and $j ; x_{i}$ and $x_{j}$ are the values (here the number of individuals) at location $i$ and $j ; W(d)$ is the sum of $w_{i j}(d)$. The local Moran's $I_{i}$ is simply the same index but for a given cell $i$,

$$
I_{i}(d)=\frac{x_{i}-\bar{x}}{\frac{1}{n} \sum_{i=1}^{n}\left(x_{i}-\bar{x}\right)^{2}} \sum_{j=1, j \neq i}^{n} w_{i j}(d)\left(x_{j}-\bar{x}\right) .
$$

Of course, Moran's $I$ is still too complicated to come up with any analytic results. For this reason, we choose a simplified global LISA index, the joincount statistics, in the following analysis (see below for detail), which allows us to reach a reasonable approximation and good analytic power of the scaling pattern of species distributions, as well as the MAUP.

\subsection{Bayesian Estimation}

\section{Pair Approximation and Join-Count Statistics}

A closely related field in spatial ecology is metapopulation ecology, which studies the spatiotemporal dynamics of binary (presence-absence) maps based on Levins' patch occupancy model [55, 56. The Levins model assumes not only the infinite number of habitat patches but also the insensitivity of colonization by distance. Since movements of most organisms are restricted in 
space and hence all patches in a large network are not likely to be equally accessible from a given patch, these assumptions seem contradictory [57]. However, what is really underlying these assumptions is that the model assumes all patches are equally connected to other patches, which is called the mean-field assumption. Although this assumption is at the heart of many ecological theories, it ignores much of what is important about the dynamics of ecological interactions. Ecological interactions such as predation, resource competition, parasitism, epidemic transmission, and reproduction often occur at spatial scales much smaller than that of the whole population [58. The dispersal and colonization of migrants in metapopulations are certainly local processes in space, and hence the distribution cannot be described by the mean-field approximation.

The most powerful approach to modeling spatially structured population dynamics and local processes in ecology are the lattice or cellular automaton models, which have been widely applied to the research of metapopulation dynamics and more general questions of spatial ecology [59]. These spatially explicit simulation models can be analyzed by a useful approach, called pair approximation, introduced to ecological research by Matsuda et al. 60. This approximation, original from statistical physics 61, 62, has been applied to many models of population dynamics of plants 63, 64, 65. These two approaches are, thus, especially designed for analyzing those spatial patterns in figure 2B, C and D.

In the spatial autocorrelation analysis by experimental and landscape ecologists, as mentioned above, within the group of LISA indices [17, the simplest index to describe the spatial pattern is the join-count statistics [21, which is also conceptually and mathematically similar to the pair-approximation (or moment approximation) approach [19, 58.

If we only consider two states, presence and absence, instead of the exact number of individuals in it, we have four states with regard to the focal cell and its randomly-chosen neighbor: an occupied cell with a neighbor that was also occupied, $q_{+/+}$; an occupied cell with an empty neighboring patch, $q_{0 /+}$; an empty cell with an occupied neighbor, $q_{+/ 0}$; an empty cell with an empty neighbor, $q_{0 / 0}$. In fact we only need two variables $\left(p_{+}, q_{+/+}\right)$to express all the other join-count statistics:

$$
\begin{gathered}
p_{0}=1-p_{+} \\
q_{0 /+}=1-q_{+/+} \\
q_{+/ 0}=\left(1-q_{+/+}\right) p_{+} /\left(1-p_{+}\right) \\
q_{0 / 0}=\left(1-2 p_{+}+q_{+/+} p_{+}\right) /\left(1-p_{+}\right)
\end{gathered}
$$

There is still an inequality controlling the balance between $p_{+}$and $q_{+/+}$, $0 \leq p_{+} \leq 1$ and $2-1 / p_{+} \leq q_{+/+} \leq 1[20$. These are the join-count statistics, the simplest indices for describing the spatial pattern observed. If $q_{+/+}>p_{+}$, we have a spatially autocorrelated population (aggregated). For the spatial 

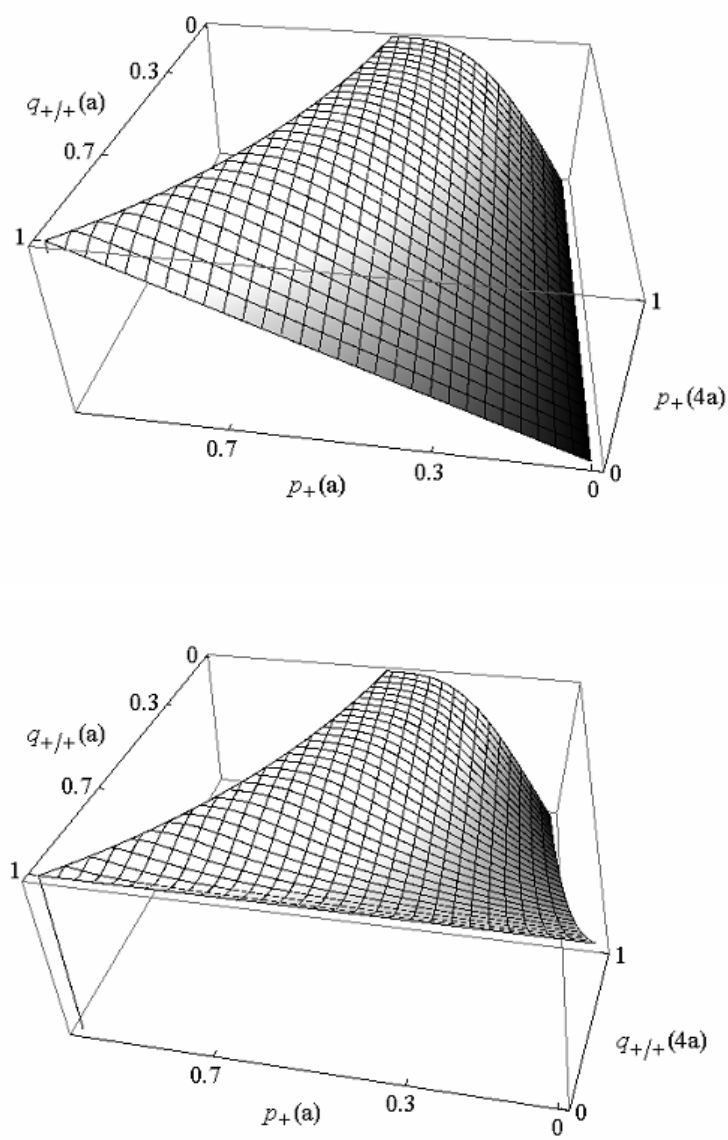

Fig. 3. The occupancy and the spatial correlation by scaling-up

pattern in figure $2 \mathrm{~B}$, we have $q_{+/+}=0.3837>p_{+}=0.2794$; for figure $2 \mathrm{C}$, $q_{+/+}=0.7589>p_{+}=0.6325$. If $q_{+/+}=p_{+}$, we have a spatially random one. The ratio $q_{+/+} / p_{+}$or the difference $q_{+/+}-p_{+}$gives the degree of spatial clustering [21, 22. Of course, the group of LISA can analyze the spatial patterns for not only the presence-absence data, and as a consequence it can provide more information on spatial autocorrelation, i.e. it can describe the clustering of abundance in samples by for example Moran' $I$ index 66. The following sections will show how we predict the occupancy in figure $2 \mathrm{C}$ and $\mathrm{D}$ with only the knowledge from figure $2 \mathrm{~B}$ using a Bayesian estimation.

\section{The Scale Problem}

Using the join-count statistics, we can scale up the sample size (grain) [6]. For a cell in figure $2 \mathrm{C}$, it is difficult to calculate the probability of presence since it could be 1,2 , or 541 individuals in the cell, which leads to a superimposed 
distribution of multiple binomial distributions 67, 68]. Since the presence probability is equal to one minus the absence probability, it will be much easier for us to calculate the absence probability first. The absence probability $p_{0}(a)$ and the correlation of two adjacent empty patches $q_{0 / 0}(a)$ with scaling up (grain from $a$ to $4 a$ ) will be,

$$
\begin{gathered}
p_{0}(4 a)=p_{0}(a) \times q_{0 / 0}(a)^{2} \times b_{0}(a) \\
q_{0 / 0}(4 a)=q_{0 / 0}(a)^{2} \times b_{0}(a)^{2}
\end{gathered}
$$

where $a$ indicates the grain size (e.g. if the grain size of figure $2 \mathrm{~B}$ is $a$, then the one of figure $2 \mathrm{C}$ will be $4 a) ; b_{0}(a)$ is the probability that a sample patch with two empty neighboring patches is absent. If we choose a Bayesian estimation (BYE) for $b_{0}(a)$, it will be,

$$
b_{0}(a)=\frac{q_{0 / 0}(a)^{2} \times p_{0}(a)}{q_{0 / 0}(a)^{2} \times p_{0}(a)+q_{0 /+}(a)^{2} \times p_{+}(a)}
$$

According to probability rules, $p_{+}=1-p_{0}$ and $q_{+/+}=1-\left(1-q_{0 / 0} p_{0} / p_{+}\right)$. In 2006, Hui, McGeoch and Warren [6] presented a formula governing the pattern of the occupancy and spatial correlation with increase of scale,

$$
\begin{gathered}
p_{+}(4 a)=1-\frac{\nabla^{4}}{\triangle} \\
q_{+/+}(4 a)=\frac{\nabla^{10}-2 \nabla^{4} \triangle^{2}+\triangle^{3}}{\triangle^{2}\left(\triangle-\nabla^{4}\right)}
\end{gathered}
$$

where $\nabla=p_{0}(a)-q_{0 /+}(a) p_{+}(a)$ and $\triangle=p_{0}(a)\left[1-p_{+}(a)^{2}\left(2 q_{+/+}(a)-3\right)+\right.$ $\left.p_{+}(a)\left(q_{+/+}(a)^{2}-3\right)\right]$.

An important result here is that the occupancy $p_{+}(a)$ and spatial correlation $q_{+/+}(a)$ will both limit to 1 with the increase of grain (figure 3 ) [18, which means that the spatial distribution of species will change from aggregation to randomness with scaling-up $\left(p_{+}(a)=q_{+/+}(a)\right)$. Using equations (4) and (5), as well as $p_{+}(a)=0.2793$ and $q_{+/+}(a)=0.3837$ for figure $2 \mathrm{~B}$, we have the occupancy and spatial correlaiton for the $20 \times 20$ resolution (figure $2 \mathrm{C}$ ), $p_{+}(4 a)=0.6672$ and $q_{+/+}(4 a)=0.6849$, closed to the obervation $\left(p_{+}(4 a)=0.6325\right.$ and $\left.q_{+/+}(4 a)=0.7589\right)$. The accurracy for occupancy (defined as 1 - Abs (Predicted - Observed)/Predicted, 6]) is 95\% and for spatial correlation is $89 \%$. Furthermore, equations (4) and (5) only have four variables: occupancies and spatial correlation at grains $a$ and $4 a$.

What makes the BYE function more powerful is that it can predict the occupancy and the spatial correlation at fine-scales using coarse-scale data. For example, under the condition of an aggregated spatial distribution $\left(q_{+/+}>p_{+}\right)$, we can solve $p_{+}(a)$ and $q_{+/+}(a)$ using $p_{+}(4 a)$ and $q_{+/+}(4 a)$, the prediction for figure $2 \mathrm{~B}$ is $p_{+}(a)=0.4129$ and $q_{+/+}(a)=0.7418$. Because fine-scale binary (presence-absence) data are more information rich 
than coarse-scale data [69], scaling-up predictions of occupancy are bound to be more accurate than those obtained by scaling-down. Other tests using Drosophilidae species across the mesocosm arena also confirm the prediction capacity of equations (4) and (5) [6]. Thus, the BYE provides a preliminary solution to the scale problem in the MAUP.

\section{The Aggregation Problem}

For the transect shape grain as in figure $1 \mathrm{C}$ and figure $2 \mathrm{D}$, we can calculate its probability for absence $p_{0}(4 a)$ and the conditional probability $q_{0 / 0}(4 a)$ according to the BYE [6],

$$
\begin{gathered}
p_{0}(4 a)=p_{0}(a) \times q_{0 / 0}(a)^{3} \\
q_{0 / 0}(4 a)=q_{0 / 0}(a) \times b_{0}(a)^{3} \\
q_{0 / 0}(4 a)=q_{0 / 0}(a)^{4}
\end{gathered}
$$

where equation (7) is the probability adjacent to the long edge and equation (8) is to the short edge. Using the similar procedure, we can have,

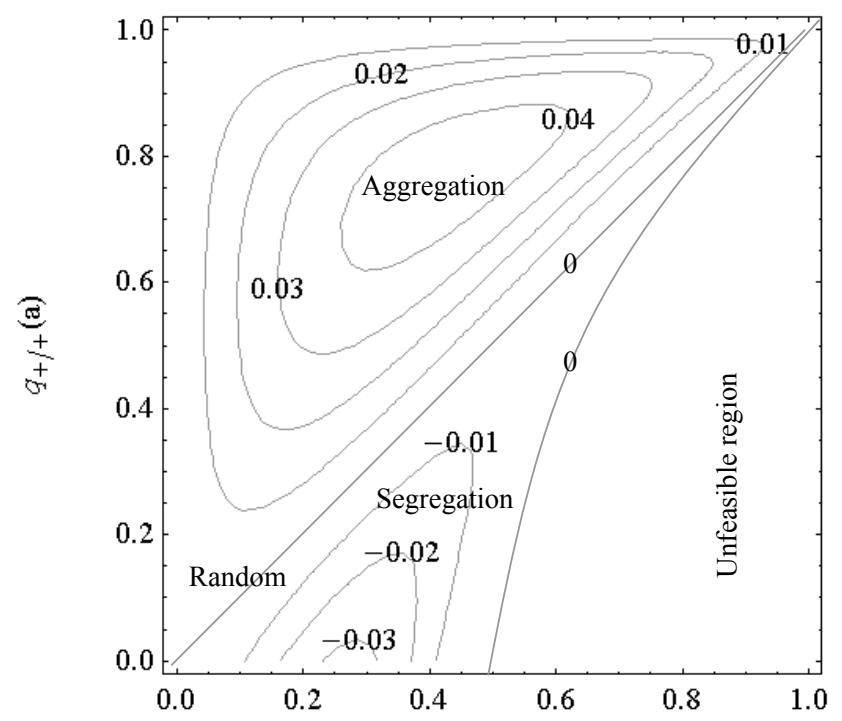

$$
p_{+}(\text {a) }
$$

Fig. 4. The contour plot of the difference between the occupancy with the transect grain (as in figure 1C) and the one with the square grain (as in figure 1B), $p_{+}(4 a)_{\text {transect }}-p_{+}(4 a)_{\text {square }}$. The unfeasible region indicates $2-1 / p_{+}>q_{+/+}$; the aggregation region indicates $p_{+}<q_{+/+}$; the segregation region indicates $p_{+}>q_{+/+}$, random indicates the line that $p_{+}=q_{+/+}$. 


$$
p_{+}(4 a)=1-\frac{\left(1-2 p_{+}(a)+p_{+}(a) \times q_{+/+}(a)\right)^{3}}{\left(1-p_{+}(a)\right)^{2}}
$$

The formula for $q_{+/+}(4 a)$ were not shown here for conciseness but can easily be obtained using equations (7) and (8). According to equation (9), we have the occupancy for figure $2 \mathrm{D}, p_{+}(4 a)=0.6823$, which is comparable to the observation $\left(p_{+}(4 a)=0.65\right)$.

A quick test of the spatial distribution pattern can be executed, by comparing the occupancy in a chessboard (as in figure 1B) with the one in a transect grain map (as in figure 1C). We find that the occupancy will be larger in a longer-perimeter sample if the spatial distribution is aggregated but will be smaller if it is segregated in samples with similar sample area or grain (see figure 4; 6]). In a real case, De Grave and Casey [70] reported the variability in density estimates of intertidal, infaunal benthos due to the shape of sampling devices. They found that the density for most intertidal macrofauna is lower in rectangular shaped samples compared to the density observed in square shaped samples with similar area, but the situation was inversed for,$\ldots$, , $, \ldots, r, \ldots, \cdots$, Bate. According to the above analysis, the reason might be that most species are aggregated in space while.$\cdots$, . . , has a segregated distribution due to its high mobility. Therefore, the BYE further provides the solution for the aggregation (zoning) problem in the MAUP.

\subsection{Scaling Patterns}

As demonstrated above, this iterative formula of the Bayesian solution calculates the species occupancy and spatial correlation after combining four neighboring cells into one new larger grain, and thus fits the understanding of species scaling pattern as a percolation process [71, 72. Criticism of the formula mainly concerns the difficulty in calculation [73, as well as its restriction of linking species distributions in a grain of $a$ only to those in a grain of $4 a$, i.e. its discrete essence. This section is intended to advance the understanding of the scaling pattern of species distribution based on the same rationale behind this Bayesian estimate. This will be achieved by generating, through induction, new formulae of species occupancy and spatial correction that rely on scale (grain size) as the only independent variable. Intriguingly, this formulae not only simplifies the Bayesian estimate, but also generates the shapes of species scaling patterns that are consistent with those from the intraspecific occupancy-abundance relationship (OAR) and the area-ofoccupancy (AOO) models. OAR describes the positive correlation between the abundance and occupancy of a species over time or across regions, is one of the most widely used descriptions of species distribution patterns [74, 72]. AOO has been found to obey a log-log linear relationship across sample scales (grain sizes) [72. Such a log-log linear AOO provides an instant link to the box-counting fractal dimension [72, and therefore has been argued to reveal the scale-invariant nature of the species distribution. 


$$
\left[\begin{array}{ccc}
\rho_{i, j} & \rightarrow & \rho_{i, j+1} \\
\downarrow & & \Downarrow \\
\rho_{i+1, j} & \Rightarrow & \rho_{i+1, j+1}
\end{array}\right] \rightarrow\left[\begin{array}{ccc}
\rho_{i, j+2} & \rightarrow & \rho_{i, j+3} \\
\downarrow & & \Downarrow \\
\rho_{i+1, j+2} & \Rightarrow & \rho_{i+1, j+3}
\end{array}\right]
$$

Fig. 5. The procedure of calculating the probability of absence in a $(2 \times 2)$-cell (left block), $p_{0}(2 \times 2)$, and the conditional probability of a randomly chosen neighbor of this $(2 \times 2)$-cell being also absent, $q_{0 / 0}(2 \times 2)$. Black arrows indicate conditional probabilities; white arrows indicate Bayesian estimates.

A spatial (presence-absence) map can be expressed by a binary matrix, $M=<\rho_{i, j}>_{m \times m}$, with the element $\rho_{i, j}$ being either + or 0 , indicating the state of the cell $(i, j)$ as either occupied or empty respectively. The dimensionality of the matrix, $m \times m$, can normally be considered as infinite. Now if we combine $n \times n$ cells together to form a new cell, the binary matrix will become $M=<\rho_{i, j}>_{m / n \times m / n}$, with $\rho_{i, j}=1$ indicating that at least one previous cell (or a sub-cell) is occupied and $\rho_{i, j}=0$ indicating that all $n \times n$ previous cells are empty. A solution to the spatial scaling of species distribution is to calculate the global density (occupancy) and the local density (spatial correlation) for various grain sizes. it can be achieved by first calculating the probability of absence $p_{0}(n \times n)$ and $q_{0 / 0}(n \times n)$, and then using the relationships between those probabilities, $p_{+}=1-p_{0}$ and $q_{+/+}=1-\left(1-q_{0 / 0}\right) p_{0} /\left(1-p_{0}\right)$ to calculate $p_{+}(n \times n)$ and $q_{+/+}(n \times n)$.

For $n=2$, given that the probability of a randomly selected cell being empty $\left(\rho_{i, j}=0\right)$ is $p_{0}$, the probability of finding two empty neighbors $\left(\rho_{i, j+1}=0\right.$ and $\left.\rho_{i+1, j}=0\right)$ is $q_{0 / 0}^{2}$ and the probability that a shared neighbor of these two absent cells is also empty $\left(\rho_{i+1, j+1}=0\right)$ be denoted as $b_{0}$, we can easily obtain $p_{0}(2 \times 2)=p_{0} \times q_{0 / 0}^{2} \times b_{0}$ and $q_{0 / 0}(2 \times 2)=q_{0 / 0}^{2} \times b_{0}^{2}$ according to the diagram in figure 5 .

For $n \geq 3$, probabilities $p_{0}(n \times n)$ and $q_{0 / 0}(n \times n)$ can be calculated according to different procedures of combining $n \times n$ cells in forming a new larger grain. This is essentially similar to the mathematical problem of the seven bridges of Konigsberg. For example when $n=3$, it is possible to calculate $p_{0}(3 \times 3)$ and $q_{0 / 0}(3 \times 3)$ using a spiral method of adding neighbors together to form a larger grain (figure $6 \mathrm{~A}$ ). Accordingly, we have $p_{0}(3 \times 3)=p_{0} \times q_{0 / 0}^{6} \times b_{0} \times g_{0}$ and $q_{0 / 0}(3 \times 3)=q_{0 / 0}^{6} \times b_{0} \times k_{0} \times g_{0}$, where $g_{0}$ is the probability that a shared neighbor of four absent cells is empty, and $k_{0}$ is the probability that a shared neighbor of three absent cells is empty. These two extra probabilities, i.e. $g_{0}$ and $k_{0}$, also need to be estimated in this spiral procedure of scaling-up. Notably, this is not the only procedure to combine nine cells together in forming larger grains. In figure $6 \mathrm{~B}$, another procedure is presented for calculating the probability of $p_{0}(3 \times 3)$ and $q_{0 / 0}(3 \times 3)$, which gives $p_{0}(3 \times 3)=p_{0} \times q_{0 / 0}^{4} \times b_{0}^{4}$ and $q_{0 / 0}(3 \times 3)=q_{0 / 0}^{4} \times b_{0}^{4} \times k_{0}$. The number of prcedures of calculating $p_{0}(n \times n)$ and $q_{0 / 0}(n \times n)$ becomes extremely large for $n>>3$ (similar to the number of Euler walks). To solve this problem 
A

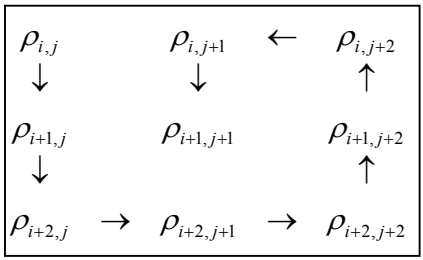

B

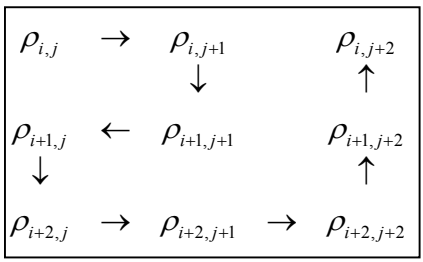

$\mathrm{C}$

\begin{tabular}{|c|c|c|c|}
\hline $\begin{array}{c}\rho_{i, j} \\
\downarrow\end{array}$ & $\begin{array}{c}\rho_{i, j+1} \\
\uparrow\end{array}$ & $\rightarrow$ & $\begin{array}{c}\rho_{i, j+2} \\
\quad \downarrow\end{array}$ \\
\hline$\rho_{i+1, j}$ & $\rho_{i+1, j+1}$ & & $\rho_{i+1, j+2}$ \\
\hline$\downarrow$ & $\uparrow$ & & $\downarrow$ \\
\hline$\rho_{i+2, j} \quad \rightarrow$ & $\rho_{i+2, j+1}$ & & $\rho_{i+2, j+2}$ \\
\hline
\end{tabular}

Fig. 6. The procedures of calculating the probability of absence in a $(3 \times 3)$-cell. Black arrows indicate the order of adding sub-cells using, $\boldsymbol{A}$ a spiral procedure, $\boldsymbol{B}$ an alternative procedure, and $\boldsymbol{C}$ a zigzag procedure. see text for detail.

and also to avoid calculating extra probabilities, a procedure is used here for calculating $p_{0}(n \times n)$ and $q_{0 / 0}(n \times n)$ according to a zigzag method of adding cells together. For example, following this zigzag procedure (figure $6 \mathrm{C}$ ), we have $p_{0}(3 \times 3)=p_{0} \times q_{0 / 0}^{4} \times b_{0}^{4}$ and $q_{0 / 0}(3 \times 3)=q_{0 / 0}^{3} \times b_{0}^{6}$. After examining several more cases (e.g. for $n=4$ and 5), formulae can easily be induced for the general case:

$$
\begin{gathered}
p_{0}(n \times n)=p_{0} \times q_{0 / 0}^{2(n-1)} \times b_{0}^{(n-1)^{2}}, \\
q_{0 / 0}(n \times n)=q_{0 / 0}^{n} \times b_{0}^{n(n-1)} .
\end{gathered}
$$

Since $n \times n$ indicates the size of the new grain (denoted as $a$ ), we have the scaling pattern of absence,

$$
p_{0}(a)=b_{0}^{a}\left(\frac{p_{0} \times b_{0}}{q_{0 / 0}^{2}}\right)\left(\frac{q_{0 / 0}}{b_{0}}\right)^{2 a^{1 / 2}},
$$




$$
q_{0 / 0}(a)=b_{0}^{a}\left(\frac{q_{0 / 0}}{b_{0}}\right)^{a^{1 / 2}} .
$$

Accordingly, we can simply get the following scaling patterns of occupancy and spatial correlation,

$$
\begin{gathered}
p_{+}(a)=1-\theta \times \beta^{2 a^{1 / 2}} \times \delta^{a}, \\
q_{+/+}(a)=p_{+}(a)+\frac{\left(\theta^{-1} \times \beta^{-a^{1 / 2}}-1\right)\left(1-p_{+}(a)\right)^{2}}{p_{+}(a)},
\end{gathered}
$$

where $\theta=p_{0} \times b_{0} / q_{0 / 0}^{2}, \beta=q_{0 / 0} / b_{0}$ and $\delta=b_{0}$ are model parameters. This scaling pattern describes species occupancy and spatial correlation as a function of the spatial scale $a$, and elucidates the percolation process of non-random structure when scaling up.

The scaling patterns of species distribution were illustrated in figure 7 . First, if we classify the spatial structure into three categories: aggregation $\left(q_{+/+}>p_{+}\right)$, randomness $\left(q_{+/+}=p_{+}\right)$and segregation $\left(q_{+/+}<p_{+}\right)$[6, 20], the category of spatial structure will not change across categories with change of scales (figure 7A), i.e. it remains in the same category. With the increase of grain $a$, occupancy $p_{+}(a)$ increases monotonously, whereas the spatial correlation $q_{+/+}(a)$ decreases at first but then increases ultimately at the same rate of $p_{+}(a)$, i.e. it converges to randomness. The watershed threshold for $q_{+/+}(a)$ changing from declining to increasing [the dashed line in figure 7A] can not be solved analytically, but an approximate numerical solution can be obtained as an ellipse, $\left(p_{+}-1\right)^{2} / 1.14^{2}+q_{+/+}^{2} / 0.89^{2}=1$, after a rotation of $\pi / 4$. This threshold is also insensitive to the initial values of $p_{0}$ and $q_{0 / 0}$. It is worth noting that the spatial correlation is not perfectly synchronized with the changing rate of occupancy $d p_{+}(a) / d a$; however generally speaking, a lower spatial correlation indeed corresponds with a higher changing rate of occupancy, i.e. the scale-dependence of occupancy becomes strong when the spatial correlation is weak [6]. Second, the overall saturated-curve-shape and the S-shape (especially obvious for those highly aggregated patterns, $\left.q_{+/+}>>p_{+}\right)$of occupancy scaling are consistent with the results from OAR models 72 . Furthermore, if the spatial structure is random, it is easy to obtain that $p_{+}(a)=1-e^{-\lambda \times a}$ (where $\lambda=-\ln (\delta)$ ), which is actually the Poisson OAR for randomness (e.g. [74, 72]). Finally, a quasi-power law holds for the scaling pattern of occupancy over about two orders of scale magnitude (figure 7B), which is consistent with a log-log linear AOO as reported in literature 75]. However, such a quasi-power law does not reflect a strict self-similar nature of species distributions. A further test on the spatial structure reveals the scale-dependence of spatial correlation (figure 7C). A power law form of AOO does not explain how those occupied cells distribute, and, therefore, does not necessarily lead to the scale-invariance (self-similarity) of the spatial distribution, i.e. the fractal objects can always lead to a power law scaling, but not vice verse. The approach presented here facilitates accurate, cost-efficient estimation of occupancy, and provides a comprehensive approach towards modelling species distributions. 

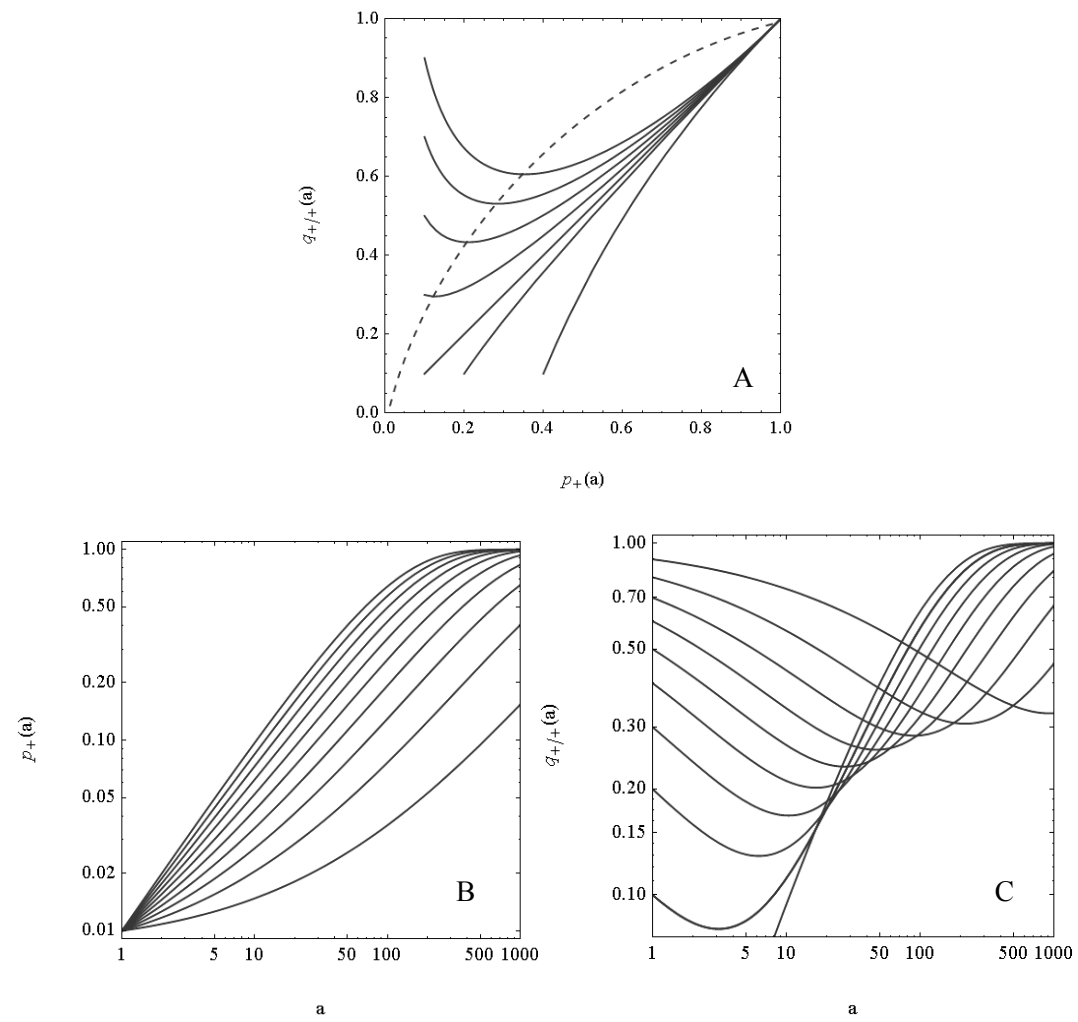

Fig. 7. Scaling patterns of species occupancy and spatial correlation. $\boldsymbol{A}$, Several trajectories of $p_{+}(a)$ and $q_{+/+}(a)$ in a parametric plot, which all lead to the topright with the increase of grain $a$. The dashed line indicates the watershed threshold of $q_{+/+}(a)$ where it changes from declining to increasing with the increase of grain a. $\boldsymbol{B}$, Scaling patterns of occupancy, with $p_{+}=0.01$ and $q_{+/+}=0,0.1,0.2, \ldots, 0.9$ (from top to bottom curves). $\boldsymbol{C}$, Scaling patterns of spatial correlation, with the same initial values as in $\boldsymbol{B}$.

\section{Conclusion}

From the above analysis, the following propositions are brought forward. (1: ) With the increase of grain size, the occupancy $p_{+}$and the spatial correlation $q_{+/+}$both increase. The BYE can largely explain the trajectories of these two variables across scales. $(2:, \ldots, \ldots, \ldots, \ldots)$ The accuracy of prediction of the BYE for scaling-up is higher than for scalingdown; the accuracy of prediction of the BYE for occupancy, $p_{+}$(first-order description of the spatial distribution), is higher than for the spatial correlation, $q_{+/+}$(second-order description of the spatial distribution). (3: , $\ldots$ $, \cdots, \cdots, \ldots, \cdots, \ldots, \ldots)$ Under the same grain size, the occupancy prediction is sensitive to irregularity (or length of the perimeter) as well as the 
spatial sturcture (the intensity of aggregation, randomness and segregation; e.g. those described by the join-count statistics). If the spatial structure is aggregated $\left(p_{+}<q_{+/+}\right)$, occupancy estimates with longer perimeter grain will have higher values. If the spatial structure is random $\left(p_{+}=q_{+/+}\right)$, occupancy estimates will not be affected by the shape of the grain. If the spatial structure is segregated $\left(p_{+}>q_{+/+}\right)$, occupancy estimates with longer perimeter grains will have higher values of occupancy than those with shorter perimeter grain. Thus, the BYE provide a preliminary solution to the presence-absence format MAUP.

The above analysis shows that it will be possible in the near future to provide a comprehensive solution to the MAUP. Studies on the scaling pattern of species distribution present a framework to the scale problem in MAUP. Hui and McGeoch 72 have reviewed different occupancy-abundance models as well as the scaling pattern of occupancy. Because such essential links between presence-absence data and the abundance (or the intensity) of the spatial patterns, those more general models, slotting them into the BYE framework, will eventually resolve the scale problem of the MAUP. Moreover, the solution to the scale problem will also benefit conservation science. Kunin [75] gave a log-log linear area-of-occupancy (i.e. the relationship between occupancy and grain size), which has been used to predict the biodiversity trend [76]. Hartley and Kunin [77] suggested that the solution to the scale problem can also be used to estimate the abundance of focal species based only on presence-absence data, or even on the occupancy alone. Such techniques will surely improve the efficiency of conservation management. Furthermore, He and Hubbell [71] have explored the effect of perimeter length of grain on the occupancy and abundance estimates. Their findings are consistent with the analysis of the aggregation problem here in the MAUP. The solution to the aggregation problem will help us to understand ecological fallacy and sampling artifacts. In conclusion, the BYE together with those advances will contribute to building a comprehensive solution to the Modifiable Areal Unit Problem.

\section{Future Directions}

Research should foucs on the following with regard to solving the MAUP in the spatial analysis. First, a scale-free index for the description of spatial patterns is urgently needed. Up to now, as outlined in this chapter, spatial statistics and indices are largely scale-dependent. Even though a few indices, such as Shannon's information and entropy index, have proclaimed to be scale-free, this is questionable under stingent testing. Furthermore, although specific scale-dependent statistics and indices might be especially valuable for case studies, they contribute little to solving the scale problem as a whole. Second, more effort should be put into finding proper measurements of irregular shapes (e.g. the width-to-length ratio here). Studies have revealed the strong correlation between the grain irregularity and the intensity of the 
zoning effect 6, 71. Fractality might shed light on this issue as its initial intention is to describe the irrigularity of fractal, self-similar objects. Finally, the MAUP is a push forward for unveiling the spatial character of natural systems rather than simply a problem. Fotheringham [78] has suggested the shifting of spatial analysis towards relationships that focus on rates of change (see also 24]): "Can we acquire information on the rate of change in variables and relationships of interest with respect to scale?" This is exactly the focus of this study, i.e. seeking the amount of change when the grain shifts from $a$ to $4 a$. Furthermore, Hui and McGeoch 72 indicated that the scale problem is eventually a percolation process. Linking with knowledge from other nonlinear sciences will surely help to overcome the MAUP in spatial analysis.

Suprisingly, the analysis in this chapter can also be used in the analysis of co-variance and association, which is not part of the MAUP. Species association belongs to the compositional variance, measuring the degree of co-occurrence and co-distribution of two species in samples [3, 5], the scaling pattern of which is closely linked with the mechnaism behind beta diversity and species-area relationship [3, 4, 5, 79. For instance, considering two species, there exist four scenarios for a randomly chosen cell: species $A$ and $B$ coexist, $P_{A \cap B}(a)$ (also called the joint occupancy); only species $A$ occurs, $P_{A \cap \bar{B}}(a)$; only species $B$ occurs, $P_{\bar{A} \cap B}(a)$; neither exists, $P_{\bar{A} \cap \bar{B}}(a)$. Note that $P_{A}(a)$ and $P_{B}(a)$ have the same meanings as $p_{+}(a)_{A}$ and $p_{+}(a)_{B}$, respectively. Similarly, we can define a postive association of species $A$ and $B$ as $P_{A \cap B}(a)>P_{A}(a) \times P_{B}(a)$, a negative association as $P_{A \cap B}(a)<P_{A}(a) \times P_{B}(a)$, and the independence of the distribution of these two species as $P_{A \cap B}(a)=P_{A}(a) \times P_{B}(a)$. This definition is also well consisitent with the study of null models for species co-occurrence [80, 81]. Therefore, the same framework developed in this chapter can also be used to calculate the scaling pattern of association and co-variance. Further exploration of the inclusion of spatial autocorrelation structure into spatial models should be productive. Indeed, the fields of spatial analysis [12, 43, and modelling [58] have developed largely independently to date. Integration of these two approaches, as demonstrated in this chapter, are likely to result in significant advances towards the development of a general spatial framework for understanding the non-randomness phenomena in nature, and surely warrants further attention.

\section{References}

1. Levin, S.A.: The problem of pattern and scale in ecology. Ecology 73, 1943-1967 (1992)

2. Plimak, L.I., Walls, D.F.: Nonclassical spatial and momentum distributions in a Bose-condensed gas. Phys. Rev. A 54, 652-655 (1996)

3. Whittaker, R.H.: Evolution and measurement of species diversity. Taxon 21, 213-251 (1972)

4. Hui, C.: On species-area and species accumulation curves: a comment on Chong and Stohlgren's index. Biol. Indic. 8, 327-329 (2008) 
5. Hui, C., McGeoch, M.A.: Does the self-similar species distribution model lead to unrealistic predictions? Ecology 89, 2946-2952 (2008)

6. Hui, C., McGeoch, M.A., Warren, M.: A spatially explicit approach to estimating species occupancy and spatial correlation. J. Anim. Ecol. 75, 140-147 (2006)

7. Openshaw, S.: The modifiable areal unit problem. Geo Books, Norwick (1984)

8. Unwin, D.J.: GIS, spatial analysis and spatial statistics. Prog. Human Geogr. 20, 540-551 (1996)

9. Burger, O., Todd, L.: Grain, extent, and intensity: the components of scale in archaeological survey. In: Lock, G., Molyneaux, B.L. (eds.) Confronting scale in archaeological: issues of theory and practice, pp. 235-255. Springer, New York (2006)

10. Taylor, L.R.: Aggregation, variance and the mean. Nature 189, 732-735 (1961)

11. Ratcliffe, J.H., McCullagh, M.J.: Hotbeds of crime and the search for spatial accuracy. Geogr. Sys. 1, 385-395 (1999)

12. Wiens, J.A.: Ecological heterogeneity: ontogeny of concepts and approaches. In: Hutchings, M.J., Jones, E.A., Stewart, A.J.A. (eds.) The ecological consequences of environmental heterogeneity, pp. 9-31. Blackwell Science, Oxford (2000)

13. Li, H., Reynolds, J.F.: On definition and quantification of heterogeneity. Oikos 73, 280-284 (1995)

14. Burrough, P.A., McDonnell, R.A.: Principles of geographical information systems. Oxford Univ. Press, Oxford (1998)

15. Perry, J.N., Liebhold, A.M., Rosenberg, M.S., Dungan, J.L., Miriti, M., Jakomulska, A., Citron-Pousty, S.: Illustrations and guidelins for selecting statistical methods for quantifying spatial pattern in ecological data. Ecography 25, 578$600(2002)$

16. Dungan, J.L., Perry, J.N., Dale, M.R.T., Legendre, P., Citron-Pousty, S., Fortin, M.J., Jakomulska, A., Miriti, M., Rosenberg, M.S.: A balanced view of scale in spatial statistical analysis. Ecography 25, 626-640 (2002)

17. Anselin, L.: Local indicators of spatial association. Geogr Analysis 27, 93-116 (1995)

18. Hui, C.: Crossing the borders of spatial analysis and modelling: a rethink. In: Kelly, J.T. (ed.) Progress in Mathematical Biology Research, pp. 170-197. Nova Science, Hauppauge (2008)

19. Sato, K., Iwasa, Y.: Pair approximation for lattice-based ecological models. In: Dieckmann, U., Law, R., Metz, J.A.J. (eds.) The geometry of ecological interactions: simplifying spatial complexity, pp. 341-359. Cambridge Univ Press, Cambridge (2000)

20. Hui, C., Li, Z.: Distribution patterns of metapopulation determined by Allee effects. Popul. Ecol. 46, 55-63 (2004)

21. Fortin, M.J., Dale, M.R.T., ver Hoef, J.: Spatial analysis in ecology. In: ElShaarawi, A.H., Piegorsch, W.W. (eds.) Encyclopedia of environmentrics, pp. 2051-2058. Wiley and Sons, New York (2002)

22. Hui, C., McGeoch, M.A.: Spatial patterns of prisoner's dillema game in metapopulations. Bull. Math. Biol. 69, 659-676 (2007)

23. Gehlke, C., Biehl, K.: Certain effects of grouping upon the size of the correlation coefficient in census tract material. J. Am. Stat. Assoc. 29, 169-170 (1934)

24. Jelinski, D.E., Wu, J.: The modifiable areal unit problem and implications for landscape ecology. Land Ecol. 11, 129-140 (1996) 
25. Fotheringham, A.S., Wong, D.W.S.: The modifiable areal unit problem in multivariate statistical-analysis. Environ. Plan A 23, 1025-1044 (1991)

26. Dorling, D.: The visualization of local urban change across Britain. Environ. Plan B 22, 269-290 (1995)

27. Amrhein, C.G.: Searching for the elusive aggregation effect - Evidence from statistical simulations. Environ. Plan A 27, 105-119 (1995)

28. Dark, S.J., Bram, D.: The modifiable areal unit problem (MAUP) in physical geography. Prog. Phys. Geogr. 31, 471-479 (2007)

29. Downey, L.: Using geographic information systems to reconceptualize spatial relationships and ecological context. Am. J. Soc. 112, 567-612 (2006)

30. Flowerdew, R., Manley, D., Steel, D.: Scales, levels and processes: Studying spatial patterns of British census variables. Comp. Environ. Urban. Sys. 30, 2143-2160 (2006)

31. Lery, B.: A comparison of foster care entry risk at three spatial scales. Subs Use Misuse 43, 223-237 (2008)

32. Sexton, K., Waller, L.A., McMaster, R.B., Maldonado, G., Adgate, J.L.: The importance of spatial effects for environmental health policy and research. $\mathrm{Hu}-$ man Ecol. Risk Ass. 8, 109-125 (2002)

33. Lembo, A.J., Lew, M.Y., Laba, M., Baveye, P.: Use of spatial SQL to assess the practical significance of the modifiable areal unit problem. Comp. Geosci. 32, 270-274 (2006)

34. Wong, D.W.S.: Spatial decomposition of segregation indices: A framework toward measuring segregation at multiple levels. Geogra. Anal. 35, 179-194 (2003)

35. Harrison, J.A., Allan, D.G., Underhill, L.G., Herremans, M., Tree, A.J., Parker, V., Brown, C.J.: The atlas of Southern African birds, BirdLife South Africa, Johannesburg (1997)

36. Fielding, A.H., Bell, J.F.: A review of methods for the assessment of prediction errors in conservation presence/absence models. Environ. Cons. 24, 38-49 (1997)

37. Kadmon, R., Farber, O., Danin, A.: A systematic analysis of factors affecting the performance of climate envelope models. Ecol. Appl. 13, 853-867 (2003)

38. Hui, C., Li, Z.: Dynamical complexity and metapopulation persistence. Ecol. Model 164, 201-209 (2003)

39. Hui, C., Yue, D.: Niche construction and polymorphism maintenance in metapopulations. Ecol. Res. 20, 115-119 (2005)

40. Levin, S.A., Grenfell, B., Hastings, A., Perelson, A.S.: Mathematical and computational challenges in population biology and ecosystem science. Science 275, 334-343 (1997)

41. Hui, C., McGeoch, M.A.: Evolution of body size, range size, and food composition in a predator-prey metapopulation. Ecol. Complex 3, 148-159 (2006)

42. Hui, C., Li, Z., Yue, D.X.: Metapopulation dynamics and distribution, and environmental heterogeneity induced by niche construction. Ecol. Model 177, 107-118 (2005)

43. Fortin, M.J., Dale, M.R.T.: Spatial analysis: a guide for ecologists. Cambridge Univ. Press, Cambridge (2005)

44. Fahrig, L., Nuttle, W.K.: Population ecology in spatial heterogeneous environments. In: Lovett, G.M., Jones, C.G., Turner, M.G., Weathers, K.C. (eds.) Ecosystem function in heterogeneous landscapes, pp. 95-118. Springer, Berlin (2005) 
45. Pacala, S.W., Levin, S.A.: Biologically generated spatial pattern and the coexistence of competing species. In: Tilman, D., Kareiva, P. (eds.) Spatial ecology: the role of space in population dynamics and interspecific interactions, pp. 204-232. Princeton Univ. Press, Princeton (1997)

46. Downing, J.A.: Biological heterogeneity in aquatic ecosystems. In: Kolasa, J., Pickett, S.T.A. (eds.) Ecological heterogeneity, pp. 160-180. Springer, Berlin (1991)

47. Morisita, M.: $I_{d}$-index, a measure of dispersion of individuals. Res. Popul. Ecol. 4, 1-7 (1962)

48. Lloyd, M.: Mean crowding. J. Anim. Ecol. 36, 1-30 (1967)

49. Bliss, C.I., Fisher, R.A.: Fitting the negative binomial distribution to biological data. Biometrics 9, 176-200 (1953)

50. Ripley, B.D.: Spatial statistics. Wiley, New York (1981)

51. Moran, P.A.P.: Notes on continuous stochastic phenomena. Biometrika 37, 17$23(1950)$

52. Geary, R.C.: The contiguity ratio and statistical mapping. Incorp. Stat. 5, 115145 (1954)

53. Perry, J.N.: Spatial analysis by distance indices. J. Anim. Ecol. 64, 303-314 (1995)

54. Perry, J.N.: Measures of spatial pattern for counts. Ecology 79, 1008-1017 (1998)

55. Levins, R.: Some demographic and genetic consequences of environmental heterogeneity for biological control. Bull. Entomol. Soc. Am. 15, 237-240 (1969)

56. Hanski, I.: Metapopulation dynamics. Nature 396, 41-49 (1998)

57. Hanski, I.: Metapopulation ecology. Oxford Univ. Press, Oxford (1999)

58. Dieckmann, U., Law, R., Metz, J.A.J.: The geometry of ecological interactions: simplifying spatial complexity. Cambridge Unive. Press, Cambridge (2000)

59. Tilman, D., Karieva, P.: Spatial ecology: the role of space in population dynamics and interspecific interactions. Princeton Univ. Press, Princeton (1997)

60. Matsuda, H., Ogita, A., Sasaki, A., Sato, K.: Statistical mechanics of population: the lattice Lotka-Volterra model. Prog. Theor. Phys. 88, 1035-1049 (1992)

61. Katori, M., Konno, N.: Upper bounds for survival probability of the contact process. J. Stat. Phys. 63, 115-130 (1991)

62. Tainaka, K.: Paradoxical effect in a three-candidate voter model. Phys. Lett. A 176, 303-306 (1993)

63. Iwasa, Y., Sato, K., Nakashima, S.: Dynamic modeling of wave regeneration (Shimagare) in subalpine Abies forests. J. Theor. Biol. 152, 143-158 (1991)

64. Harada, Y., Ezoe, H., Iwasa, Y., Matsuda, H., Sato, K.: Population persistence and spatially limited social interaction. Theor. Popul. Biol. 48, 65-91 (1994)

65. Harada, Y., Iwasa, Y.: Lattice population dynamics for plants with dispersing seeds and vegetative propagation. Res. Popul. Ecol. 36, 237-249 (1994)

66. Moran, P.A.P.: Notes on continuous stochastic phenomena. Biometrika 37, 17$23(1950)$

67. Hui, C., McGeoch, M.A.: A self-similarity model for the occupancy frequency distribution. Theor. Popul. Biol. 71, 61-70 (2007)

68. Hui, C., McGeoch, M.A.: Modeling species distributions by breaking the assumption of self-similarity. Oikos 116, 2097-2107 (2007)

69. McGeoch, M.A., Gaston, K.J.: Occupancy frequency distributions: patterns, artefacts and mechanisms. Biol. Rev. 77, 311-331 (2002) 
70. De Grave, S., Casey, D.: Influence of sample shape and orientation on density estimates on intertidal macrofauna. J. Marine Biol. Assoc. UK 80, 1125-1126 (2000)

71. He, F., Hubbell, S.P.: Percolation theory for the distribution and abundnce of species. Phys. Rev. Lett. 91, 198103 (2003)

72. Hui, C., McGeoch, M.A.: Capturing the "droopy-tail" in the occupancyabundance relationship. Ecoscience 14, 103-108 (2007)

73. Meynard, C.N., Quinn, J.F.: Predicting species distributions: a critical comparison of the most common statistical models using artificial species. J. Biogeogr. 34, 1455-1469 (2007)

74. Holt, A.R., Gaston, K.J., He, F.: Occupancy-abundance relationships and spatial distribution: a review. Basic Appl. Ecol. 3, 1-13 (2002)

75. Kunin, W.E.: Extrapolating species abundance across spatial scales. Science 281, 1513-1515 (1998)

76. Wilson, R.J., Thomas, C.D., Fox, R., Roy, D.B., Kunin, W.E.: Spatial patterns in species distributions reveal biodiversity change. Nature 432, 393-396 (2004)

77. Hartley, S., Kunin, W.E.: Scale dependency of rarity, extinction risk, and conservation priority. Cons. Biol. 17, 1559-1570 (2003)

78. Fotheringham, A.S.: Scale-independent spatial analysis. In: Goodchild, M.F., Gopal, S. (eds.) Accuracy of spatial databases, pp. 221-228. Taylor and Francis, London (1989)

79. Scheiner, S.M.: Six types of species-area curves. Global Ecol. Biogeogr. 12, 441-447 (2003)

80. Bell, G.: The co-distribution of species in relation to the neutral theory of community ecology. Ecology 86, 1757-1770 (2005)

81. Gotelli, N.J., Graves, G.R.: Null models in ecology. Smithsonian Institution Press, Washington (1996) 